\title{
THE
}

$11-24-2014$

\section{Successful Strategies for Discharging Medicaid Nursing Home Residents with Mental Health Diagnoses to the Community}

Skye N. Leedahl

University of Rhode Island, skyeleedahl@uri.edu

Rosemary K. Chapin

Carrie Wendel

Beth Anne Baca

Leslie K. Hasche

Follow this and additional works at: https://digitalcommons.uri.edu/hdf_facpubs

The University of Rhode Island Faculty have made this article openly available.

Please let us know how Open Access to this research benefits you.

This is a pre-publication author manuscript of the final, published article.

Terms of Use

This article is made available under the terms and conditions applicable towards Open Access

Policy Articles, as set forth in our Terms of Use.

\section{Citation/Publisher Attribution}

Skye N. Leedahl, Rosemary K. Chapin, Carrie Wendel, Beth Anne Baca, Leslie K. Hasche \& Grace W. Townley (2015) Successful Strategies for Discharging Medicaid Nursing Home Residents With Mental Health Diagnoses to the Community, Journal of Social Service Research, 41:2, 172-192, DOI: 10.1080/ 01488376.2014 .972012

Available at: http://dx.doi.org/10.1080/01488376.2014.972012

This Article is brought to you for free and open access by the Human Development and Family Science at DigitalCommons@URI. It has been accepted for inclusion in Human Development and Family Science Faculty Publications by an authorized administrator of DigitalCommons@URI. For more information, please contact digitalcommons-group@uri.edu. 


\section{Authors}

Skye N. Leedahl, Rosemary K. Chapin, Carrie Wendel, Beth Anne Baca, Leslie K. Hasche, and Grace W. Townley 


\section{Title}

Successful Strategies for Discharging Medicaid Nursing Home Residents with Mental Health

Diagnoses to the Community

\section{Journal}

Journal of Social Service Research

\section{Authors}

Skye N. Leedahl, PhD, ${ }^{1}$ Corresponding Author

Rosemary K. Chapin, $\mathrm{PhD}^{2}$

Carrie Wendel, $\mathrm{MA}^{2}$

Beth Anne Baca, MSW ${ }^{2}$

Leslie K. Hasche, PhD, MSW ${ }^{3}$

Grace W. Townley, LMSW ${ }^{4}$

${ }^{1}$ University of Rhode Island, Departments of Human Development \& Family Studies and Political Science, Aging and Health Faculty Fellows Program, 2 Lower College Road, Kingston, RI 02881

${ }^{2}$ University of Kansas, School of Social Welfare, Center for Research on Aging and Disability Options (CRADO), 1545 Lilac Lane, Lawrence, KS 66044

${ }^{3}$ University of Denver, Graduate School of Social Work, 2148 S. High Street, Denver, CO 80208

${ }^{4}$ Hearthstone Alzheimer Care, 305 West End Avenue, New York, NY 10023 


\section{Funding}

This work was supported in part through a contract with the Kansas Department for Aging and

Disability Services (KDADS) (Contract 10-KU-002).

\section{Acknowledgements}

The authors acknowledge the leadership and staff of Kansas Department for Aging and

Disability Services who aided in securing the data and supported the project. We also thank

Julie Sergeant, Roxanne Rachlin, and Mary Zimmerman for their contributions to the study.

\section{Correspondence}

Please address all correspondence to Skye N. Leedahl, PhD, University of Rhode Island, 2

Lower College Road, Kingston, RI 02881. Email: skyeleedahl@ uri.edu. Phone: 401-874-4567 


\begin{abstract}
The state and federal push to transition Medicaid residents from nursing homes to the community calls for effective discharge strategies targeted to residents' diverse needs. This exploratory, mixed methods study utilized the Minimum Data Set (MDS) to describe demographics, health characteristics, and transition patterns of Kansas Medicaid residents with mental health diagnoses who were discharged from nursing homes between 2005-2008. Discharged residents $(n=720)$ had multiple comorbidities, and over half remained in the community following their first nursing home event. In-depth interviews with nursing home staff $(n=11)$ explored successful discharge strategies. Successful strategies support an ecological approach to meeting individual, family, organizational, and community needs. This includes creating/sustaining a culture of discharge, inclusion of informal and formal community supports in the discharge process, proactively addressing physical environment needs, and assisting individuals and their family members in managing physical and mental health conditions. Findings suggest that policies in the areas of pre-admission screening, caregiver support, and revised Medicaid reimbursement are needed to better support continuity of care and promote discharge for nursing home residents with complex care needs. Future research could examine individual and family perspectives on the discharge process and track outcomes when transitioning between settings.
\end{abstract} Keywords: Nursing home, discharge, mental health diagnoses, informal supports, community resources 
Successful Strategies for Discharging Medicaid Nursing Home Residents with Mental Health Diagnoses to the Community

Most frail elders and adults with disabilities would like to continue to live in the community (Bartels, Miles, Dums, \& Levine, 2003; Bayer \& Harper, 2000; Feldman, Oberlink, Simantov, \& Gursen, 2004), and research findings support the cost effectiveness of providing long term care services in the community versus in a nursing home (NH) (Chapin, Baca, Macmillan, Rachlin, \& Zimmerman, 2009a; Grabowski, 2006). As a result, many policy efforts have emerged to support discharge from NHs to community-based settings. In addition, the prevalence of mental health diagnoses for individuals in NHs is higher than in community-based settings, with estimates as high as 58 to 68 percent (Kerber, Dyck, Culp, \& Buckwalter, 2005; Mechanic \& McAlpine, 2000; U.S. Department of Health and Human Services, 1999), and the number of NH residents with mental health diagnoses continues to rise (Bartels \& Drake, 2005). Existing research documents concerns with mental health services provided in the NH (Grabowski, Aschbrenner, Rome, \& Bartels, 2010) and concerns that NHs are being used as substitutes for inpatient psychiatric care (Simon, Lipson, \& Stone, 2011). However, little is known about discharging this population specifically. Therefore, understanding if and how NH residents with mental health diagnoses transition to the community is needed.

In recent decades, federal and state governments have prioritized transitioning $\mathrm{NH}$ residents to the community, and some research has focused on examining populations that discharge and best practices for ensuring safe transitions (e.g., Watts, 2013). However, while individuals who move into a NH have significant physical health needs and functional limitations, those who also have a mental health diagnosis, including anxiety, bipolar, and/or schizophrenia, and receive Medicaid have compounded needs and challenges. For example, Muramatsu and Goebert (2011) reported a number of challenges associated with treating those 
with mental health concerns in a $\mathrm{NH}$, including concerns regarding dangerousness, the need for greater staff attention, and lack of trained staff as well as difficulties with accessing psychiatric support and rehospitalization. Further, Meador et al. (2011) found that older adults receiving Medicaid services are less likely to be discharged from a NH to a community setting due to limited available resources. Due to these challenges, some state policy makers and practitioners (Chapin et al., 2010; Chapin et al., 2009b) questioned whether this population can discharge, how often discharge occurs for this population, and importantly, what works in helping them discharge. The dual purpose of this study was (1) to describe a sample of NH residents in Kansas with mental health diagnoses who use Medicaid and have discharged to the community; and (2) to identify successful discharge strategies for transitioning this population from the NH to community settings. This study provides valuable insights that can help inform various public policies as well as NH staff, community service providers, and mental health professionals by extending the literature on best practices for discharge for those with mental health diagnoses.

\section{Literature Review}

\section{Policy Background}

An emphasis on more accessible and available community-based services as an alternative to $\mathrm{NH}$ care emerged in federal and state policies during the late 1970s and early 1980s. An important step in this direction occurred with the development of Medicaid Home and Community Based (HCBS) waivers in 1983, which allowed Medicaid funds, once restricted to institutional care, to provide community-based long term services and supports (LTSS). These efforts were bolstered in the late 1990s with Nursing Home Transition Demonstration Grants awarded to twelve states by the Centers for Medicare and Medicaid Services (CMS) (Kasper \& O’Malley, 2006). These grants, which had few restrictions, were used to overcome barriers 
people faced when transitioning from NHs and to create community supports. The Deficit Reduction Act of 2005 created the Money Follows the Person (MFP) Program, which approved $\$ 1.75$ billion to encourage transitions from $\mathrm{NHs}$ to the community and the further development of Medicaid HCBS (Lipson et al., 2007). The MFP program has been extended as part of the 2010 health reform legislation with 44 states participating (CMS, 2014).

The MFP program is designed to reduce dependence on NH care and strengthen the ability of Medicaid programs to provide care to people who choose to live in the community by increasing HCBS availability, eliminating policy barriers that restrict the use of Medicaid funds, and improving quality assurance (CMS, 2014; Lipson et al., 2007). The MFP program meets these objectives by changing state policies to allow Medicaid funding to "follow the person to the setting of his or her choice" (Lipson \& Williams, 2009, p. 2) and by providing support services in the community for up to 365 days after discharge (Wenzlow \& Lipson, 2009). However, recent research indicates that MFP funds may be serving individuals with fewer needs more successfully than those with higher needs (Lim \& Irvin, 2013). The authors conclude that more research on discharge transitions and reinstitutionalization in the MFP program is needed, especially for those with complex care needs.

Importantly, the MFP program complements other federal policies meant to improve care for LTSS customers with mental health needs. For example, under the Omnibus Budget Reconciliation Act of 1987 (OBRA 1987), the Pre-Admission Screening and Resident Review (PASRR) required states to screen individuals with any issues related to mental illness or intellectual or developmental disabilities prior to admission to a NH. This process is completed in order to ensure that individuals with these conditions are placed in a facility that can meet their specialized needs. Although designed to improve institutional care, recent work has 
documented how some states also use the PASRR process to divert and/or transition NH residents with mental illness into community-based care (O’Connor, Ingle, \& Wamback, 2012). Additionally, the 1999 Olmstead decision of the U.S. Supreme Court established that individuals with disabilities, including mental health diagnoses, have the right to receive community-based care whenever feasible (Kasper \& O’Malley, 2006). However, because it is challenging to find the necessary resources for those with mental health needs in addition to other needs, fewer individuals with mental illness tend to transition to the community (Wenzlow \& Lipson, 2009).

\section{Supporting Transitions Between Nursing Home and Community Settings}

This study builds on the broader literature on predictors of discharges and readmissions between NH and community settings and on practice strategies used within transition programs. For example, a study completed by Gassoumis, Fike, Rahman, Enguidanos, and Wilber (2013) compared short-stay (90 days or less) versus long-stay (91 to 365 days) residents who transitioned to the community in Southern California over a three-year period. The strongest predictor of community discharge for short-stay residents was having a support person supporting the decision to discharge. Very little predicted discharge for long-stay residents, though having cancer and being severely cognitively impaired reduced the odds of discharge. Related to the present study, mental health diagnoses did not predict discharge for either shortstay or long-stay residents. In terms of risk factors for readmission into NHs within one year of discharge, a study of New Jersey's nursing home transition program identified that $72.6 \%$ of

participants remained in the community (Howell, Silberberg, Quinn, \& Lucas, 2007). This study identified that (1) being male, single, and dissatisfied with one's living situation; (2) living with others; and (3) falling within 8 to 10 weeks after discharge were significant predictors of NH readmission. Unfortunately, this latter study did not include mental health variables. 
While the federal grants provide much needed support for transitioning individuals from NHs to the community, the specific strategies used by NH staff and other professionals to successfully discharge NH residents are not yet well known. Some transition programs across the country have been successful in discharging NH residents to the community, and these programs' strategies vary widely (Kasper \& O’Malley, 2006). Typical components of these transition programs include coordination or case management to help consumers access housing, a fund to pay up-front costs for community living, and a method for identifying those interested in transitioning and likely to succeed in community living (Eiken, 2003). Recently, Meador et al. (2011) reported on an intervention called Project Home that helps older adults living in a NH transition to a community setting. Services provided in this program include: an initial in-depth analysis; the development of transition plan that includes all aspects of care including how to do laundry or fill a prescription; and intensive case management aimed at gaining access to services in the community.

Although these details on practice strategies are helpful, research continues to identify additional unmet needs of individuals who transitioned from $\mathrm{NH}$ to the community. A study identified unmet needs of individuals who transitioned from Connecticut's HCBS program to NHs using administrative data, care manager notes, and focus groups (Robison, Shugrue, Porter, Fortinsky, \& Curry, 2012). Study findings indicated that acute health problems, mental health issues, and family caregiver stress were inadequately addressed. Further unmet needs included the lack of home care providers available on nights and weekends, limited transportation resources, and the scarcity of supportive housing options.

Similar to other research, the present study focuses on understanding transitions between $\mathrm{NH}$ and the community for individuals in one state. It advances this literature by identifying 
characteristics and transition patterns for a population with complex needs (i.e., individuals with mental health diagnoses who utilize Medicaid) over a three-year period across the state of Kansas, while also detailing specific practice strategies used by NH staff members as part of their daily practice for discharging Medicaid residents with mental health diagnoses. Such information may help NH staff increase discharge rates for Medicaid residents who experience compounded challenges, such as physical and mental health limitations.

\section{Research Aims}

In 2009, the Kansas Department for Aging and Disability Services requested an exploratory study of NH discharge for residents with mental health diagnoses who use Medicaid. The state was interested in informing their MFP program efforts for this population and their mental health policies and practices for NH residents. Initially the university research team conducted interviews with a random sample of Kansas state NH staff and other key stakeholders involved in discharging this population to the community. This preliminary study indicated that some NH staff had safety concerns about discharging this population to the community, as they questioned the availability of adequate community-based supports. Additionally, NH staff stated that residents with mental health needs often have "ping-pong" patterns of living in the facility and community, meaning they often go in and out of NHs multiple times (Chapin et al., 2009b). However, there was no existing research documenting numbers or patterns of NH discharge for Medicaid beneficiaries with mental health diagnoses. Furthermore, both state agency and NH staff were eager to learn about specific strategies that have led to successful discharges for those with mental health diagnoses across the state.

Therefore, this study builds on the input of these key stakeholders. Study research questions were: 1) How many Medicaid consumers with mental health diagnoses discharged 
from the NH to a community setting in Kansas between 2005-2008, and what are their demographic and health characteristics and transition patterns (i.e., NH to community)?; and 2) What specific strategies have NH staff used to successfully discharge this population to community settings?

\section{Theoretical Framework}

This study utilized an ecological model as a framework for understanding discharge strategies at multiple levels (e.g., individual, community, society). The ecological perspective focuses on how people and their environments affect each other and recognizes that many systems interact to influence outcomes (Bronfenbrenner, 1979; McLaren \& Hawe, 2005) (e.g., NH discharge to the community). The ecological perspective highlights various components of people's environments including social networks, organizations, communities, and public policies (Richard, Potvin, Kishchuk, Prlic, \& Green, 1996; Sanders, Fitzgerald, \& Bratteli, 2008). The use of this perspective emphasizes older adults' ability to manage everyday concerns and provides a basis for recognition that discharge is a multi-dimensional occurrence best understood as the outcome of transactions overtime between the person and environment (Green \& Sullivan, 2004).

In addition, the use of an ecological model helped to consider how the various systems interact to enable $\mathrm{NH}$ discharge and to identify discharge strategies that capitalize on people's strengths to overcome systemic barriers. Figure 1 provides a pictorial representation of the ecological model used for this project. In this model, $\mathrm{NH}$ discharge to the community was the outcome. The study focused on the identified five systems, or levels, of potential influence on the outcome.

Insert Figure 1 about here 
At the first level are the individual characteristics and attributes that influenced discharge for those re-entering the community. These include gender, mental health diagnosis, and income status. Focusing on this first level helped identify strategies based on specific resident attributes and circumstances. At the second level, strategies that addressed the physical environment were considered. The physical environment includes features of the community, neighborhood, and physical structure of the home (e.g., urban/rural, available housing, specialized group living, continuing care retirement communities (CCRCs)). Third, strategies that identified, utilized, and sustained informal supports were emphasized. Informal supports for community living include assistance and encouragement from family, friends, and neighbors. Fourth, strategies that addressed service needs in the community (e.g., home health, mental health, transportation, meals) were identified. Finally, strategies for addressing structural forces that mitigated against discharge (e.g., lack of resources) as well as strategies that are influenced by social policies (e.g., HCBS waiver, Older Americans Act) were considered.

\section{Methods}

This study utilized a concurrent mixed methods approach to answer research questions. Secondary quantitative data were analyzed to determine the characteristics of those discharging from $\mathrm{NH}$ and their transition patterns. To learn about successful discharge strategies, qualitative interviews with NH staff across Kansas who helped successfully discharge NH residents with mental health diagnoses to the community were conducted. Qualitative and quantitative research methods complemented each other, which provided data triangulation and contributed to the identification of practice and policy implications for increasing discharge rates. The university Institutional Review Board approved the study, including the use of human subjects. 


\section{Quantitative Methods to Examine Resident Characteristics and Transition Patterns}

The Minimum Data Set (MDS) 2.0 provided comprehensive information about older

adults at admission to NHs, during quarterly and annual reassessments, and upon discharge. The MDS is a resident assessment instrument administered to all residents in Medicare or Medicaid certified NHs (Morris et al., 1990) and is used for program administration and policy purposes. These data were used to identify the characteristics of a sample of residents who entered into and discharged from Kansas NHs (i.e., skilled nursing facilities and long-term care units within hospitals) between July 2005 through June 2008. The total sample included individuals who: 1) were admitted to an $\mathrm{NH}$ for the first time on or after July 1, 2005 and discharged to the community (indicated by the following responses to item R3a: Private home/apartment with no home health services, Private home/apartment with home health services, or Board and care/assisted living/group home) at least once between July 1, 2005 through June 30, 2008; 2) had a mental health diagnosis of anxiety (indicated by a 'yes' response to item I1dd), bipolar disorder (indicated by a 'yes' response to item I1ff) and/or schizophrenia (indicated by a 'yes' response to item I1ff) as indicated on the admission assessment; and 3) were enrolled in Medicaid (indicated on item AA7). While depression is one of the most common mental health diagnoses among NH residents (Bagchi, Verdier, \& Simon, 2009) and does potentially impact NH discharge, depression was not included as a primary mental health diagnosis due to known concerns with the validity of the MDS depression measures (Simmons et al., 2004).

To identify transition patterns, each individual's initial admission and initial discharge assessment that occurred between July 1, 2005, and June 30, 2008 was identified, and then additional admissions and community discharges for each individual were tracked through December 31, 2008. In this count, data were included from July-December 2008 to ensure at 
least six months' follow-up time for all adults in the study. A community discharge was defined as an admission to a $\mathrm{NH}$ followed by a transition from a $\mathrm{NH}$ to a community-based setting (i.e., private home, assisted living/residential care home) for any length of time. Descriptive statistical analyses were completed using SPSS 17.0 software.

\section{Qualitative Methods to Explore Discharge Strategies}

In-depth, semi-structured interviews were conducted with eleven NH staff to answer the second research question on successful discharge strategies for this population. MDS data, previously described, was used to identify all NHs in the state of Kansas that had discharged multiple (at least four) Medicaid residents with a mental health diagnosis to the community during the study timeframe $(n=29)$. There were $280 \mathrm{NHs}$ in Kansas at the time. Because NHs in some rural regions did not have multiple discharges for this population, NHs from these areas $(n=9)$ that had at least one discharge during the study period were included to ensure representation of NHs across the state. This purposive sampling strategy provided opportunities to talk with various discharge staff from NHs that enable the most discharges, and therefore could be considered experienced with discharging Medicaid residents with mental health diagnoses.

To recruit participants, recruitment letters were sent to the administrators of eligible NHs, and staff members made follow-up phone calls explaining the purpose of the study and study procedures. Ten administrators referred NH discharge staff who agreed to participate in the study ( $40 \%$ response rate). The most common reason for declining participation in the study was related to staff workload issues. Participants were recruited and interviews were conducted until reaching the point of saturation, meaning no new information was being gathered and the sample represented a wide range of $\mathrm{NH}$ characteristics across the state. This resulted in a total sample of 11 staff representing $10 \mathrm{NHs}$. 
The NHs represented all areas of the state, with seven of the NHs being in more urban areas and three in more rural areas. Interviews were conducted in-person $(n=4)$ or over the phone $(n=6)$ between February and April of 2010. All discharge staff interview participants $(n$ $=11)^{1}$ were female. Ten worked in social services, primarily as the Directors of Social Services, and one person was the Director of Nursing. Training varied among participants, with five of the participants being licensed social workers, three having received social services designee training, and three having other degrees and training (e.g., nursing). The average number of years of employment at the current $\mathrm{NH}$ was 10.4 years (range $=6$ months to 27 years). The length of time in the position with discharge responsibilities varied from 6 months to 20 years, with an average of 9.5 years. Finally, the overall length of time spent working in long term care was an average of 14.6 years, and the range was from 1.5 years to 28 years.

One researcher completed all ten interviews. The interviews ranged in length from 21 minutes to 77 minutes ( $M=47$ minutes), and were all recorded and transcribed. To gather information about strategies used to successfully discharge Medicaid NH residents with mental health diagnoses to the community, the semi-structured interview guide consisted of questions covering 1) general information about the NH staff members (e.g., position title, length of time working in the facility, duties related to discharge), 2) strategies considered most effective for discharging the overall target population and those with specific physical and mental health comorbidities 3) specific strategies used for transitioning residents with multiple transitions from the NH to the community (i.e., ping-pong patterns), and 4) suggestions for increasing discharge rates.

Qualitative data analysis began immediately following the completion of each interview. The interviewer also took field notes on key interview content and interviewer 
impressions. Emergent themes were noted in research memos, which also informed revisions to the interview guide. A four-person project team conducted open-coding of the transcripts assisted by Atlas.ti qualitative software. The transcripts were reviewed and recoded throughout multiple layers of coding, thus strengthening the reliability of the coding schemas.

\section{Findings}

\section{Demographic and Health Characteristics}

Medicaid residents with mental health diagnoses who discharged from NHs in Kansas were found to be a very diverse group. Between July 1, 2005 and June 30, 2008, there were 720 people who received Medicaid; had a diagnosis of anxiety disorder, bipolar disorder, or schizophrenia; had both a base admission and base discharge assessment; and discharged to a community setting. This represented $20.6 \%$ of 3,488 individuals using Medicaid who discharged to the community during the study timeframe. Within the sample of 720 people discharging with a mental health condition, 69.0\% $(n=497)$ had a diagnosis of anxiety disorder, $21.8 \%(n=157)$ had a diagnosis of bipolar disorder, and 20.4\% $(n=147)$ had a diagnosis of schizophrenia. There were 75 individuals (10.4\%) with more than one mental health diagnosis. For example, 27 individuals in the total sample (3.8\%) reported both an anxiety disorder and bipolar disorder. Individuals who discharged from NHs ranged in age from 20 to 100 years at the time of their initial admission, with an average age of 64.7 years $(S D=15.4)$. Approximately $70 \%$ of the sample was female. In addition, the majority (88.5\%) were White, non-Hispanic. This is consistent with gender and racial/ethnic characteristics of the $\mathrm{NH}$ population in Kansas (CMS, 2012) and in the United States (Kaye, Harrington, \& LaPlante, 2010).

Within the sample, individuals spent an average of 83 days in the $\mathrm{NH}$ before discharging to a community setting. The length of stay for individuals ranged from less than a day to 2.7 
years $\left(997\right.$ days). ${ }^{2}$ Nearly $75 \%(n=539)$ of these individuals discharged within 90 days (considered short-stays), and 181 (25.1\%) discharged after more than 91 days in the $\mathrm{NH}$ (considered long-stays). Almost 96 percent of the discharges $(n=690)$ occurred within one year of admission to the $\mathrm{NH}$. Nearly half (43.2\%) lived alone prior to $\mathrm{NH}$ entry, and 500 individuals (69.4\%) had a support person positive towards discharge at the time of admission. These findings are similar to a study completed on transition patterns of short-stay versus long-stay $\mathrm{NH}$ residents (Gassoumis et al., 2013).

In addition to demographic characteristics, multiple physical and cognitive health characteristics of individuals at the time of their admission assessment were examined. As shown in Table 1, approximately one in eight Medicaid individuals who fit the study criteria and were discharged from the $\mathrm{NH}$ to a community setting had Alzheimer's disease or another dementia diagnosis ( $n=97,13.5 \%)$. In addition, 265 individuals (36.8\%) had diabetes mellitus. Additional comorbidities and cognitive status of individuals at the time of their admission assessment are presented in Table 1. The number of medications for each person ranged from 0$46(M=13.4, S D=5.6)$.

Insert Table 1 about here

\section{Transition Patterns}

Through the analysis, 376 individuals who experienced only one discharge from the $\mathrm{NH}$ to the community during the study period and 239 individuals who experienced multiple transitions were identified. ${ }^{3}$ Multiple transitions were defined as those who experienced one or more $\mathrm{NH}$ reentries after their initial $\mathrm{NH}$ discharge. In contrast to the perception that this 
population "ping-pongs" in and out of NHs many times, more than half of the sample (61.1\%) remained in the community consistently after discharging. Of the group who experienced multiple transitions, about half experienced one discharge and one re-entry. Interestingly, there were two study participants who had 7-8 discharges and $\mathrm{NH}$ re-entries during the study timeframe of 3 years. Thus, while "ping-pong" patterns are not common, they did occur among a small minority of residents with mental health diagnoses.

\section{Successful Discharge Strategies}

NH discharge staff identified many strategies, across all levels of the ecological model, for helping to successfully discharge Medicaid residents with physical and mental health comorbidities. Key strategies included creating an institutional culture that values and supports $\mathrm{NH}$ discharge, working to support informal and formal resources that will be used in the community, taking proactive steps to identify safe housing in the community, and preparing residents and their support persons for managing their health in the community.

Creating and supporting a culture of discharge. A key characteristic of NHs that had a successful record of discharging individuals with physical and mental health comorbidities was that discharge was valued and supported as an important goal. To support this goal, many facilities set up discharge policies and procedures that were integrated throughout an individual's NH stay. Upon admission, these discharge staff made a point to learn whether the individual is interested in discharge, as discussed by an experienced social worker from a large facility:

I think one of the primary things that we have to do is initially sit down with the resident and the family from the beginning, and outline what their personal goals are going to be as far as discharge. We've written discharge plans for several residents, though, that maybe at that time, we didn't think that they could go home. And they can surprise 
you.... It takes a long time to set up a discharge plan, but if we know from the get-go that that's their plan, that gives us a lot more time to think through all the options and to explore what's going to be available for them. So I think having that conversation initially is really important.

Beginning discharge planning and goal setting as soon as possible after admission was deemed beneficial for ensuring sufficient time to establish and work towards discharge goals. This may be especially important for residents with greater functional and health needs and for those who are long-stay (i.e., more than three months) residents, as recognized by a social worker from a medium sized facility:

The discharge plan begins at the beginning for everyone. The ones that are here shortterm are usually a lot higher functioning initially ... than the ones that are in here longterm and discharged. But we have a lady here right now that's been here, she admitted here in September, and she's going home at the end of this month [March].

These previous two quotes also indicate the wide diversity of residents who are able to discharge, and how early discharge planning can help overcome stereotypes that certain types of $\mathrm{NH}$ residents may not be suitable for discharge. Ideally, it seemed that discharge planning was person-centered and laid out specific steps and progress that should be made towards reaching the end goal of successful community tenure. NH staff found that setting discharge goals empowered residents and their family members to work toward making discharge possible and encouraged rehabilitation progress.

Many of these facilities have standardized their discharge practices, thus better ensuring that all residents have equal opportunity to work towards discharge goals. Regularly scheduled team meetings, which include the resident, their support persons, and interdisciplinary NH staff, 
was one standardized policy approach, as explained by a social worker from a continuing care retirement community:

And we have care plan meetings with the residents and the families. And those are done every 12 weeks for each resident here in long term care. When they're ready to be discharged, we always have a discharge meeting prior...a week or two before...just to make sure the family and the resident understands what our concerns might be, and they can keep us up-to-date on the progress they've made and home improvements and services.

The use of a standardized discharge form that can be personalized to each resident was another approach, as described by a social service director from a large facility:

This (discharge form) is something that I developed... I think that a big part of discharge planning is education. Families and residents don't know what services are available to them. They don't know how to get them. . . So I try to be as concise as possible on this form with the typical things that might be set up when they discharge.

Discharge forms can include special instructions for self-care from the interdisciplinary $\mathrm{NH}$ team, such as nurses, therapists, and dietitians, as well as referral and contact information for community resources. NH staff stressed the importance of ensuring information on the form is easy to understand by discharging residents and their support persons, such as avoiding use of medical jargon and acronyms. While standardized discharge procedures can help ensure that discharge remains an institutional goal and residents have equitable access to discharge planning, $\mathrm{NH}$ discharge staff also stressed the importance of flexible policies that adapt to meet individual discharge needs. 
NH staff members who have helped successfully discharge Medicaid residents with mental health diagnoses recognized the diversity of residents' support needs, and therefore included a variety of personnel throughout these discharge procedures. Successful discharge planning employed a team approach, with NH discharge staff consulting various personnel, such as social workers, physical and/or occupational therapists, speech therapists, physicians, nurses, dietary aides, mental health professionals, and community-based services professionals. Teamwork was described as essential for ensuring 1) progress towards recovery is being made while in the $\mathrm{NH}$ and 2) proper supports and arrangements are available once in the community. This point was detailed by a social worker from a large facility:

I think the critical piece here in our building is that [we] really [have] a team approach ... it's about involving the whole team. And the more people who have a vested interest in this person returning home, the more successful it's going to be. It's about involving every member of our interdisciplinary team and getting their input, and then making them a valued part of the process.

Finally, some NH discharge staff initiated follow-up contact with recently discharged residents, as indicated by a social service director from a large facility:

And then we always, of course, make routine follow-up calls after a couple of weeks of discharge, just to make sure that they've contacted anyone that they were supposed to contact or that we thought would be helpful ... A lot of times, I get kind of general questions... It's amazing. Sometimes people won't call you, but if you call them, then the questions kind of start to come out. And you know, we don't ever want them to think that just because they're discharged doesn't mean - you know, they're not technically our resident, but we'd be more than happy to get them pointed in the right direction for resources. 
In sum, these NH discharge staff believed that discharge was possible and were supported by institutions that also valued discharge. They have also worked towards establishing discharge policies and procedures that are incorporated throughout an individual's NH stay.

Including formal and informal supports in the discharge process. In discussing what they considered the most effective strategy for ensuring successful discharge from the NH to the community, NH staff members emphasized it was vital to include both formal and informal supports through discharge planning. Their discharge strategies focused on connecting individuals to formal community services and assisting informal supports, as explained by the director of nursing from a small facility:

[Those] most successful would be [those] that have the support needed to return to the community, and by support I mean they have either family members that are available to check on them daily or some type of home health or mental health [care to] make sure they are set up with appointments and follow-up with appointments.

Early on, NH staff members worked with the resident to identify and solicit the help and support of informal support persons (e.g., family members or friends, neighbors, and/or religious group members available on a regular basis), as discussed by a social worker from a large facility:

Knowing what kind of support they have in the community is real important. Do they have family that can check on them a couple times a week? Do they have someone that calls them every day? Do they have a neighbor that comes by and takes care of this or that? How do they get to their medical appointments?

They also worked directly with informal support persons serving as caregivers to ensure they have resources available to them, as indicated by a social service director from a large facility: 
That caregiver often feels a lot of guilt if they're not able to provide the type of care they need at home. I don't do the same thing every time [to help them]. It's pretty individualized. I have a lot of support group information that I give out for caregivers. I have a list of caregiver support groups in the area.

This strategy was identified as an especially important strategy for ensuring caregivers can provide anticipated support, which contributes to a successful discharge and helps to avoid $\mathrm{NH}$ re-entry for the person using Medicaid. Specifically, NH staff noted that residents and caregivers should be made aware of Alzheimer's/dementia support groups in the area, for these can greatly reduce stress. Also, staff stated notifying the caregiver of day programs and respite care can give the caregiver time to relieve stress, restore energy, and promote balance in life, thereby increasing community tenure for the person who discharged from the $\mathrm{NH}$.

Furthermore, staff discussed that it is important to recognize that residents and their caregivers may have different ideas, expectations, and goals for discharge, as shared by a social service director from a large facility:

You'd be surprised how many times the [resident] and the family member aren't wanting the same thing.

Therefore, NH staff members suggested taking extra time to listen to what both the person and caregivers (often family members) are saying, as indicated by a social service director from an urban facility:

Occasionally, I think 'this is probably going to be a little bit more complicated than most.' If I develop a good relationship with the family and residents, sometimes I can do a little mediation.... We circle around as a team and get really involved with families, get to know them, provide that one-on-one. They become more trusting. Therefore, they seem to open up with some things we don't really always want to know, but it does help. 
Understanding family situations can help align discharge goals and helps families become involved with discharge as they are willing and able. Although getting to know families and family situations takes time and effort, doing so helped in ensuring successful, safe discharge for Medicaid residents with mental health diagnoses.

Turning to formal services, discharge staff members stated that helping residents enroll and utilize HCBS waiver services was particularly critical for discharging this population. Community Mental Health Centers (CMHCs) were also widely recognized as a vital resource for NH discharging residents with mental health diagnoses. Of particular importance, these formal community service programs may be able to provide a case manager to work with the person in the community. Service providers such as Area Agencies on Aging (AAAs), Community Independent Living Centers (CILs), and Aging and Disability Resource Centers (ADRCs) also serve as a vital source of information about community resources, as expressed by a social service director from a continuing care retirement community:

Everybody who I've discharged has had a referral to AAA in one way or anotherwhether it be through case management or through the Explore Your Options (book), or getting help setting up their Medicare (Part) D plan. I think that's a central place for our residents to get information.

NH staff found that having thorough knowledge about available community supports was important. Engaging in activities that built relationships and networks with community professionals who provide Medicaid and/or mental health services was important in making successful referrals, as indicated in the following quotes from two experienced social workers: Hands on, one-on-one, calling people (other professionals), getting acquainted, making friends, being sure they know who you are and you know who they are, is probably the most helpful thing that we do ... the old saying is, it's not what you know, it's who you 
know. It's absolutely true, because if you need something and you don't know where to go, you can usually call an acquaintance, friend, that you've made in another agency that you've helped with something and say, I really need your help with this, and sometimes be able to get assistance that you might not have been able to get before.

This strategy was recommended to help ensure individuals who discharge are able to access needed community supports in a timely, efficient manner, which can be particularly challenging for those who utilize Medicaid and have mental health diagnoses.

According to those interviewed, it is also beneficial to begin incorporating formal services into discharge planning as soon as possible, in order to better promote continuity of care across residential and community settings. With each transition, there is a risk of service lapse and thus, increased anxiety. When feasible, $\mathrm{NH}$ discharge staff initiated contact between the resident and formal support persons prior to discharge, as shared by a social service designee from a small facility:

This one person in particular that I'm thinking of, this person had a caseworker [from the Community Mental Health Center]. The caseworker would come in once a week and talk with her and take her out into the community. They would go shopping, you know, gradually re-introducing them to the community ... which really works for the best, because they're panicky when they've been in such a controlled environment.

Yet, community providers cannot bill for services that NHs are expected to provide while the person is an $\mathrm{NH}$ resident, according to Medicaid rules regarding duplication of services. Nursing homes are required to ensure residents have access to needed physicians, therapy, and mental health services. Therefore, it is not always possible to start services with community-based providers prior to discharge. This can be particularly frustrating in the case of mental health 
care, as some NH discharge staff felt that individuals would be more likely to continue mental health services if they were able to establish a relationship with a community-based mental health provider prior to discharge. This may also delay the start of HCBS services, as noted by an experienced social service director:

The biggest barrier there is they can't get the services as soon as they go home, [meaning] the day they go home, because they have to actually be discharged from the facility to be coded over for the Home and Community Based Service Waiver. It can also be difficult to find community-based medical providers who accept Medicaid, due to low reimbursement rates, as explained by a social service director from a continuing care retirement community:

It's also been kind of concerning and difficult to establish primary care physicians for some of our residents. A lot of physicians aren't accepting people whose primary is Medicare and second is Medicaid. And there's really limited dental care for them as well. So that's been a concern for our discharge planning.

Despite these structural barriers, resourceful NH discharge staff still made successful referrals. They also took advantage of charitable or faith based services that may be able to fill in formal service gaps. For instance, some made referrals to donation closets that have walkers, wheelchairs, and other adaptive equipment available, and others helped residents to secure assistance with home modifications or securing household supplies from fellow church members.

In sum, these staff took many proactive steps to aid informal supports and set up formal services, in recognition of the value of these supports for successful community tenure for individuals with complex physical and mental health needs. 
Proactively addressing physical environment needs. NH staff members stated that meeting residents' preferences for community living was of utmost importance for successful discharge. In identifying suitable community housing options with the person discharging, staff considered individual circumstances and qualifications, such as physical and mental ability, income or monetary support, and availability of informal support. Housing options most often discussed were private apartments or homes, subsidized apartments, or assisted living facilities. Some NH staff found it beneficial to encourage a person who is discharging to move into a more resource-rich area if the proper resources (e.g., access to mental health services) were not available in their original community, as may be the case in rural communities.

These staff also recommended conducting a team evaluation of the discharge location along with the person and their informal support persons, in order to make safety and psychosocial recommendations, as explained below by a social worker from an urban facility: Everybody that goes home is offered the opportunity to have us go [do a home evaluation]. It's not mandatory that they go. We have had occasions where families have declined that option. But most of our families are more than willing. And with every discharge, we (discharge staff) go and then our therapy team (physical, occupational, and speech therapists) as well as a nurse. So it's really prioritized for all of the different disciplines. These professionals then made recommendations for individuals and caregivers within the context of the discharge location, as indicated by a social services director from a large facility: I request that the therapists that are going out to see them in their home. They make recommendations for how they could rearrange it to make it safer for them to get through the house. Things, easy things, like maybe you don't want a rug here because it makes you more likely to trip and break your other hip. Or, you know, you might need to widen 
this doorway so you can get your wheelchair into the toilet. Occupational therapists, especially, do a good job at that. That's kind of their specialty.

These proactive steps to ensure a safe home environment for discharging residents were discussed as important for reducing the occurrence of "ping-pong" readmission patterns.

Managing physical and mental health conditions. In the NH environment, residents have many of their physical and mental health care needs managed by an interdisciplinary team of professionals. However, upon return to the community, the individuals and their caregivers will have greater responsibility for managing their conditions. Failure to adhere to physical or mental health regimens presumably leads to crisis situations that put a person back into the NH. Yet, this can be avoided with proper education and support. Therefore, NH discharge staff recognized the importance of working closely with the residents and their caregivers to better ensure their capacity for managing physical and mental health needs once in the community.

First and foremost, this involved education on individuals' physical and mental health conditions, including characteristics of the condition, related behaviors, and treatment regimens, as indicated by a social worker with over 10 years of experience:

[We provide information] so that they understand what to expect because frequently the medical profession doesn't do a very good job explaining over all the diagnoses, behaviors associated with those, medications that they're on, why they're on them, how they might interact with each other. The thing that would offer a level of comfort [is] to know this is not unusual behavior.

Because not adhering to treatment recommendations can lead to NH re-entry, NH staff also emphasized providing information to residents and family members on the importance of 
adhering to treatment regimens as well as the possible consequences of non-adherence, as addressed by a social worker from a small facility:

[How to] increase compliance? When we work with [residents in physical] therapy, we sit down and talk about why [the therapy is important]. Sometimes it's just a matter of patient education. Be it the social work, therapy, or nursing or usually a combination of us, it usually just comes down to education.

NH staff found that education empowered residents and their family members to take charge of their situation, which contributed to increased adherence. Education on physical and mental health conditions was deemed the most helpful strategy for avoiding NH re-entries.

A number of NH staff members discussed the importance of showing residents and caregivers how to complete important critical medical treatments and therapies. NH discharge staff members found it was helpful to have a nurse demonstrate to residents and/or caregivers how to perform a health care task, watch them try it on their own, and then provide recommendations and suggestions to strengthen their skills. The following example, from a social worker at a rural facility, relates to physical therapy and the use of equipment, but could also be applied to other tasks related to physical health conditions, such as giving insulin, changing an oxygen tank, or using a feeding tube:

If we have residents here that are working in [physical] therapy, a lot of times the family and the friends will go to the therapy session to watch what the therapist is doing. Especially if they're being discharged and they're sending [the resident] home with some sort of instructions on how to continue doing therapy in the home. Also, to see how they're doing with their transfers or especially if they're using a piece of equipment that's 
different than they've used before, whether it's a wheelchair or a walker or a cane or something like that.

Specific to mental health, a number of NH staff talked about helping family members learn about specific diagnoses, treatments, and associated behaviors, thus easing questions from informal support persons such as, "Why is he/she being so difficult?" This issue was discussed by a registered nurse from a smaller $\mathrm{NH}$ :

Mental health training [would help with discharge]. Probably just some basic awareness of what to look for as far as what kinds of behaviors are displayed with people that have bipolar or schizophrenia.

NH staff suggested that being honest and forthright about mental health conditions and behaviors with individuals and family members helps them be realistic about planning for needed community-based services, such as psychiatric or respite care.

Finally, in recognition of the link between mental and physical health, these successful $\mathrm{NH}$ discharge staff also recommend non-medical approaches to maintaining health and wellbeing, as indicated by a social worker from a large, rural facility:

We know that physical health and emotional health go hand-in-hand, and so it's important for them to be able to get out and do things they're used to doing and not isolate. And so we try and make sure they have those connections too.

These NH staff often drew from holistic background training in social work or nursing.

In sum, successful NH discharge staff worked closely with individuals and their support persons to provide education and training on physical and mental health conditions and self-care, thus helping to increase compliance with physical and mental health regimens once in the community. 
Overall, the strategies discussed above operate across multiple levels of the ecological model. At the individual level, person-centered discharge planning takes into account individual physical and mental health needs and personal preferences for care. Based on unique individual needs, realistic goals were established and residents and their families were put in the driver's seat. Informal supports and formal services were integrated throughout the discharge process. The interdisciplinary $\mathrm{NH}$ team also worked to identify suitable housing and to make safety adaptations, thus addressing the physical environment. Finally, social programs such as Community Mental Health Centers and Medicaid HCBS waivers provide essential resources and support for community tenure, although reimbursement policies sometimes pose barriers to continuity of care. The desire to live in the community is a contemporary societal norm, and these staff persons and their facilities also valued this goal. Successful NH staff knew community and policy resources well, and took many creative and proactive approaches to support successful discharge and community tenure. In contrast to previously identified concerns about the ability to safely and successfully discharge Medicaid residents with physical and mental health comorbidities, these staff demonstrated strong commitment to and success in working towards discharge for $\mathrm{NH}$ residents with complex needs.

\section{Discussion and Implications}

Increasingly, state Medicaid programs are prioritizing discharge from NHs to community settings in order to save costs, provide care to individuals who need services in the most integrated setting appropriate to their needs, support older adults preference to reside in the community, and meet requirements of the Affordable Care Act. The aim of this study was to inform state policy initiatives (e.g. MFP program) and current practices regarding discharge for NH residents with mental health diagnoses who utilize Medicaid. Based on descriptive data, 
study findings provide evidence that residents of all ages with mental health diagnoses discharge from the NH to the community. Over half of the residents had only a single transition out of the NH during the three year study time period. The diversity in this group of individuals (i.e., with anxiety, bipolar, and/or schizophrenia diagnoses and a variety of physical health conditions) who discharged from the NH highlights the potential for increasing discharge among this population. Indeed, about $20 \%$ of all Medicaid residents who discharged from NHs during the study period had a mental health diagnosis. Discharging Medicaid NH residents with mental health diagnoses can be very challenging, but is a worthwhile and realistic goal. This population prefers to live in the community (Nishita, Wilber, Matsumoto, \& Schnelle, 2008), and community long term care is often more cost effective than NH care (Chapin et al., 2009a). Documenting that successful transitions can and do occur in many instances may help to assuage those policy makers and NH staff who initially are skeptical of discharge initiatives for Medicaid beneficiaries with mental health diagnoses.

From qualitative interviews with NH staff who have helped to discharge those with mental health diagnoses, discharge strategies that fit within in four key categories were identified: 1) creating an institutional culture that values and supports NH discharge, 2) working to support informal and formal resources that will be used in the community, 3) taking proactive steps to identify safe housing in the community, and 4) preparing residents and their support persons for managing their health in the community. Recognizing that individuals who discharge have a wide variety of personal and health characteristics, and yet most individuals transition only once, may help encourage future discharges for this population. Identifying a balance between formal services and informal supports in the community is crucial, and adjustments can be made based on geographic location. Furthermore, this research demonstrates 
that $\mathrm{NH}$ staff can play an important role in supporting discharge when they believe this is a realistic and attainable goal, and therefore, develop practice-based strategies to support this goal.

\section{Practice Implications}

There are numerous discharge strategies across all ecological levels for ensuring persons with mental health diagnoses receive the supports they need in the community, including for those with complex mental and physical health needs. Based on interviews with NH staff members who have successfully aided in discharging this population, $\mathrm{NH}$ administrators should consider how these and other strategies for discharge can become part of standard operating procedures in their NHs. Key applied lessons from the study include:

- Create a culture among NH staff in which discharge is treated as a desirable goal for all types of residents. For example, providing success stories to NH staff members and community agency professionals about individuals with mental health diagnoses who have discharged could help overcome misperceptions that it is not safe to discharge residents with both physical limitations and mental health diagnoses. Further, providing specific discharge strategies, as outlined in this article, could help increase discharge rates.

- Develop discharge planning procedures and integrate discharge planning throughout the resident's NH stay. This can include learning about individual's goals for discharge upon admission, outlining steps towards this goal, holding regular team meetings, and sending residents home with information and resources.

- Actively involve interdisciplinary teams, residents, and their support persons in the discharge process. Regular team meetings and a well-designed discharge form can help coordinate team efforts. Physicians, nurses, therapists, and dieticians can help 
individuals meet and plan for their physical health needs, and social work knowledge is key in tapping into local resources and mental health support.

- Encourage discharge staff to network and attend trainings to gain necessary information and resources for connecting discharging residents with supports, particularly Medicaid providers who may be difficult for the residents to access without assistance.

- Work with individuals who are discharging to develop a system of informal and formal supports prior to discharge. Ensure that these supports can be sustained after discharge through education and referrals. Follow-up with residents who have returned to the community to ensure that further referrals and information are not necessary.

- Promote person and family-centered planning by taking the time to identify individual goals and preferences for discharge. Understand how each person's unique needs, strengths, and personal circumstances shape their abilities to discharge safely into the community. Educate residents and their family members on their specific physical and mental health conditions and make recommendations for their home environment.

Although this study specifically examined discharge patterns and strategies for individuals on Medicaid with physical and mental health comorbidities, these strategies can be useful for discharging a wide range of individuals.

\section{Policy Implications}

Similar to an approach used in New Jersey's discharge program (Eiken, 2003), one strategy for starting discharge planning upon $\mathrm{NH}$ admission is to have state staff or another independent agency (e.g., AAAs, CILs, ADRCs) complete pre-admission assessments (e.g., PASRR assessment) for new NH residents that go beyond assessing the minimum information required by law. Although PASRR is traditionally used to identify appropriate NH placement for 
those with mental health or developmental disability needs, this required assessment also provides an opportunity to collect additional information, such as living preferences, on a consistent basis. In Kansas, the mandated PASRR is embedded within a larger assessment, the Client Assessment, Referral, and Evaluation (CARE). The CARE is required of all state residents seeking $\mathrm{NH}$ admission, and therefore provides an opportunity to educate residents about community-based LTSS options and document their preferences. Pre-admission assessments can help alleviate the burden on NH staff members and encourage early discharge planning, particularly if the assessment includes goals and there are effective procedures for sharing pre-admission assessment results between the designated independent agency and the NH.

Based on these findings and a review of state discharge programs, an expansion of supports for informal caregivers is recommended. Discharge staff mentioned the importance of the National Family Caregiver Support Program (NFCP), administered by AAAs in Kansas, as informal supports can be apprehensive about caring for a discharged individual. This was also recognized in Wisconsin in which Centers for Independent Living provided success stories to informal support persons and connected them to individuals and their caregivers who had already made successful transitions (Eiken, Stevenson, \& Burwell, 2002). Respite care (which can be funded by the NFCP or HCBS waivers) can also be beneficial in relieving caregiver burden and better supporting caregivers' long-term ability to provide care. Although all 50 states offer some form of respite care, this benefit is inconsistent, and many state program administrators cite limited respite options as a leading contributor to unmet family caregiver needs (Feinberg, Newman, Gray \& Kolb, 2004). 
Family caregivers are recognized as a vital resource for helping individuals with disabilities and other complex health conditions maintain community residence (Commission on Long Term Care, 2013; Direct Service Workforce Resource Center, 2011; Feinberg et al., 2004). Caregivers are expected to perform increasingly complex medical care tasks (Reinhard, Levine \& Samis, 2012), which warrants additional training and support in their caregiving role. Therefore, they need education on the physical and mental health conditions of the discharging individual, as well as instructions on any health-related tasks they are expected to perform. This was also commonly recognized as an important caregiver need by state program administrators (Feinberg et al., 2004). In a summit convened by CMS about caregiver issues, the need for additional investments into evidence-based training for caregivers was identified as an important goal for better supporting caregivers (The Direct Service Workforce Resource Center, 2011). As demonstrated above, successful NH discharge staff recognized the need to include caregivers in resident therapy and discharge planning. The federal Commission on Long Term Care (2013) recently recommended that CMS adopt policies to ensure inclusion of caregivers in LTSS care plans. This research on successful NH discharge strategies highlights the importance of these state and federal initiatives to improve caregiver supports.

LTSS policies can be improved to provide better continuity of health providers across care settings. NH discharge staff felt that having residents begin a relationship with providers (such as with mental health therapists or case managers) prior to discharge better ensures that these supports will actually be used once the individual is in the community. However, Medicaid policies may not allow for a duplication of services that are to be provided in the $\mathrm{NH}$, which often prevents community provider-patient relationships from being established prior to discharge. While a duplication of services can be wasteful, policies that allow overlap of 
institutional and community providers who provide transitional services for residents anticipated to discharge into the community, are recommended. Similarly, as noted by Meador et al. (2011), a key component to their successful discharge intervention was staff's ability to ensure services were arranged prior to discharge from the $\mathrm{NH}$, to closely monitor and repeatedly follow-up with individuals who discharged, and confirm that individuals were receiving needed services once in the community. Such policies may save money in the long run by ensuring better follow-up care and thus reducing costly NH readmissions.

Further, Medicaid reimbursement policies should be revisited in an effort to ensure widespread availability of healthcare and long term care providers. As discussed above, some NH staff struggled with finding medical and dental providers to meet the needs of discharging residents, and this may be especially difficult in rural regions. The Affordable Care Act partially and temporarily addresses this issue by requiring that Medicaid payments to primary care physicians be the same as Medicare payment rates in 2013 and 2014 (The Kaiser Commission on Medicaid and the Uninsured, 2013).

\section{Study Limitations and Future Research}

A number of study limitations are recognized. The examination of NH discharge patterns was limited by the data available, including the inability to include residents with depression in the study sample. Funding limitations precluded the use of a comparison group when examining transition patterns, such as NH residents without a mental health diagnosis or NH residents not on Medicaid. Further, it would have been helpful to have data on mortality in examining the pattern groups, so the study could have tracked rate of deaths in the community. Additionally, this study was limited to the experience of one Midwestern state. Its replication in other states would likely result in an expansion of the known successful strategies that could be used to 
increase NH discharge for this population of older adults. Future research is needed on this topic across states, which would help in understanding which states have been particularly successful in discharging those using Medicaid with mental health diagnoses from the NH to community settings.

Further research is needed to examine discharge from the perspective of individual residents and family members. Future research is also needed to track continuity of care and outcomes when moving between $\mathrm{NH}$ and community services. This would help to better understand possible lapses in care and unmet needs. Although ping-ping patterns were found to be rare, they do exist and likely account for a disproportionate share of LTSS expenditures, thus further research would aid in better understanding factors that lead to multiple transitions. Finally, future research could develop and test an intervention that utilizes the successful strategies identified to discharge a larger number of NH residents with mental health diagnoses.

\section{Conclusion}

The promotion of community-based long term supports and services has increased in recent decades, propelled by the preferences of Medicaid beneficiaries as well as by state and federal concerns about cost and quality of institutional care. This study provides a unique contribution to the literature in that it utilized a mixed methods approach to analyze MDS data to understand $\mathrm{NH}$ discharges to the community for a population with complex needs, as well as conducted interviews with a purposive sample of NH staff members who have assisted the study population in discharging to the community. Findings from this study indicate that successful transitions to the community do occur, even for individuals with mental health diagnoses of anxiety, bipolar, and schizophrenia who rely on Medicaid to pay for NH care. Despite their diverse health needs and personal circumstances, the majority of individuals who returned to 
community living after their initial $\mathrm{NH}$ admission did not re-enter the $\mathrm{NH}$ during the study timeframe. In fact, only a few individuals transitioned in and out of the NH multiple times, despite concerns by $\mathrm{NH}$ staff that this was the common pattern of $\mathrm{NH}$ transitions for this population (Chapin et al., 2009b).

Study interviews with NH staff who completed successful transitions documented that an institutional culture promoting discharge for all individuals, and the ability of NH staff to work effectively with informal and formal supports in order to meet the diverse needs of residents after discharge, are important for supporting successful community discharge. Additionally, focusing on safe housing and successfully preparing residents and support personnel to meet their physical and mental health needs in the community can help ensure that individuals will not need to return to a $\mathrm{NH}$ setting soon after discharge. In addition to documenting discharge patterns, this article also provides insights into how discharges can most effectively be accomplished from the point of view of NH staff in rural as well as urban areas, who have been instrumental in making them happen. While individually the strategies identified may be a part of daily practice for many staff members, utilizing the discharge strategies documented in this study collectively could help to increase the numbers of successful discharges for residents with mental health diagnoses. Policy makers, practitioners, and mental health advocates can use this research to press for policies and programs that support discharge and transitions to the community for people with mental health diagnoses who may not have been considered promising candidates for $\mathrm{NH}$ discharge in the past. Future research could utilize lessons learned from this study and other research to develop interventions that incorporate successful strategies as well as tracks outcomes and potential long term cost savings. 
NURSING HOME DISCHARGE STRATEGIES 


\section{References}

Bagchi, A.D., Verdier, J.M., \& Simon, S.E. (2009). How many nursing home residents live with a mental illness? Psychiatric Services, 60(7), 958-964.

Bartels, S. J., \& Drake, R. E. (2005). Evidence-based geriatric psychiatry: An overview. Psychiatric Clinics of North America, 28(4), 763-784.

Bartels, S. J., Miles, K. M., Dums, A. R., \& Levine, K. J. (2003). Are nursing homes appropriate for older adults with severe mental illness? Conflicting consumer and clinician views and implications for the Olmstead decision. Journal of the American Geriatrics Society, 51(11), 1571-1579.

Bayer, A.H., \& Harper, L. (2000). Fixing to stay: A national survey of housing and home modification issues. Washington, DC: AARP Independent Living Program.

Bronfenbrenner, U. (1979). The ecology of human development: Experiments by nature and design. Cambridge, MA: Harvard University Press.

Centers for Medicare and Medicaid Services (CMS). (2012). Retrieved on March 28, 2014 from http://www.cms.gov/Medicare/Provider-Enrollment-andCertification/CertificationandComplianc/downloads/nursinghomedatacompendium_5 08.pdf

Centers for Medicare and Medicaid Services (CMS). (2014). Retrieved on January 6, 2014 from http://www.medicaid.gov/Medicaid-CHIP-Program-Information/ByTopics/Long-Term-Services-and-Support/Balancing/Money-Follows-the-Person.html

Chapin, R.K., Baca, B., Macmillan, K., Rachlin, R., \& Zimmerman, M. (2009a). Residential outcomes for nursing facility applicants who have been diverted: Where are they 5 years later? The Gerontologist, 49(1), 46-56. 
Chapin, R., Leedahl, S.N., Baca, B.A., Sergeant, J.F., Nickerson, R.L., Zimmerman, M.K., ... Townley, G.W. (2009b). Transitioning nursing facility residents with Medicaid and mental health needs to the community. Lawrence, KS: University of Kansas School of Social Welfare.

Chapin, R.K., Leedahl, S.N., Baca, B., Sergeant, J.F., Rachlin, R., Townley, G.W., . . Loeb, K.G. (2010). Increasing nursing facility discharge for older Kansans who use Medicaid and have mental health diagnoses. Lawrence, KS: University of Kansas School of Social Welfare.

Commission on Long Term Care. (2013). Report to the Congress. Washington, DC: Author.

Direct Service Workforce Resource Center. (2011). Building capacity and coordinating support for family caregivers and the direct service workforce: Common goals and policy recommendations emerging from the CMS Leadership Summit on the direct service workforce and family caregivers. Washington, DC: Author.

Eiken, S. (2003). Community Choice: New Jersey's Nursing Home Transition Program. (Prepared for DHHS Office of the Assistant Secretary for Planning and Evaluation, Office of Disability, Aging and Long-Term Care Policy, Medstat Research and Policy Division). Washington, DC: U.S. Government Printing Office.

Eiken, S., Stevenson, D. \& Burwell, B. (2002). The homecoming project: Wisconsin's nursing home transition demonstration. Ann Arbor, MI: The Medstat Group, Inc. Retrieved on March 28, 2014 from http://aspe.hhs.gov/daltcp/reports/2002/WItrans.htm

Feinberg, L.F., Newman, S.L, Gray, L \& Kolb, K. (2004). The State of the States in Family Caregiver Support: A 50-State Study. San Francisco, CA: Family Caregiver Alliance. 
Feldman, P. H., Oberlink, M. R., Simantov, E., \& Gursen, M. D. (2004). A tale of two older Americans: Community opportunities and challenges. New York: Center for Home Care Policy and Research, Visiting Nurse Service of NY.

Gassoumis, Z. D., Fike, K. T., Rahman, A. N., Enguidanos, S. M., \& Wilber, K. H. (2013). Who transitions to the community from nursing homes? Comparing patterns and predictors for short-stay and long-stay residents. Home Health Care Services Quarterly, 32(2), 75-91.

Grabowski, D.C. (2006). The cost-effectiveness of noninstitutional long-term care services: Review and synthesis of the most recent evidence. Medical Care Research and Review, 63(1), 3-26.

Grabowski, D. C., Aschbrenner, K. A., Rome, V. F., \& Bartels, S. J. (2010). Review: Quality of mental health care for nursing home residents: A literature review. Medical Care Research and Review, 67(6), 627-656.

Greene, R. R., \& Sullivan, W. P. (2004). Putting social work values into action: Use of the ecological perspective with older adults in the managed care arena. Journal of Gerontological Social Work, 42(3/4), 131-150.

Howell, S., Silberberg, M., Quinn, W.V., \& Lucas, J.A. (2007). Determinants of remaining in the community after discharge: Results from New Jersey's Nursing Home Transition Program. The Gerontologist, 47(4), 535-547.

Kaiser Commission on Medicaid and the Uninsured. (2013). Medicaid: A Primer. Author: Washington D.C.

Kasper, J., \& O'Malley, M. (2006). Nursing home transition programs: Perspectives of state Medicaid officials. Washington, DC: Kaiser Commision on Medicaid and the Uninsured. 
Kaye, H.S., Harrington, C., \& LaPlante, M.P. (2010). Long-term care: Who gets it, who provides it, who pays, and how much? Health Affairs, 29(1), 11-21.

Kerber, C. S., Dyck, M. J., Culp, K. R., \& Buckwalter, K. (2005). Comparing the Geriatric Depression Scale, Minimum Data Set, and primary care provider diagnosis for depression in rural nursing home residents. Journal of the American Psychiatric Nurses Association, 11, 269-275.

Lim, W. \& Irvin, C.V. (2013). Disparities in post-transition outcomes by level of care needs among former nursing home residents. Cambridge, MA: Mathematica Policy Research.

Lipson, D., Gruman, C., Schimmel, J., Colby, M., Denny-Brown, N., Peterson, S., \& Williams, S.R. (2007). Money Follows the Person Demonstration Grants: Summary of state MFP program applications. Washington, DC: Mathematica Policy Research, Inc.

Lipson, D. J., \& Williams, S. R. (2009). The National Evaluation of the Money Follows the Person (MFP) Demonstration Grant Program, Reports from the Field, 2, Implications of state program features for attaining MFP transition goals. Princeton, NJ: Mathematica Policy Research, Inc.

McLaren, L., \& Hawe, P. (2005). Ecological perspectives in health research. Journal of Epidemiology Community Health, 59, 6-14.

Meador, R., Chen, E., Schultz, L., Norton, A., Henderson, C., \& Pillemer, K. (2011). Going home: Identifying barriers to nursing home discharge. Care Management Journals, 12(1), 2-11.

Mechanic, D., \& McAlpine, D. D. (2000). Use of nursing homes in the care of persons with severe mental illness: 1985 to 1995. Psychiatric Services, 51(3), 354-358. 
Morris, J.N., Hawes, C., Fries, B.E., Phillips, C.D., Mor, V., Katz, S., ... Friedlob, A.S. (1990). Designing the National Resident Assessment Instrument for nursing homes. The Gerontologist, 30(3), 293-307.

Muramatsu, R.S., \& Goebert, D. (2011). Psychiatric services: Experience, perceptions, and needs of nursing facility multidisciplinary leaders. Journal of The American Geriatrics Society, 59, 120-125.

Nishita, C.M., Wilber, K.H., Matsumoto, S., \& Schnelle, J.F. (2008). Transitioning residents from nursing facilities to community living: Who wants to leave? Journal of The American Geriatrics Society, 56, 1-7.

O'Connor, D., Ingle, J.S., \& Wamback, K.N. (2012). Leveraging the PASRR process to divert and transition elders with mental illness from nursing facilities. Journal of Aging \& Social Policy, 23(3), 305-322.

Olmstead v. L.C., 527 U.S. 581 (1999).

Omnibus Budget Reconciliation Act of 1987. 42 C.F.R. §483.120(c).

Reinhard, S., C. Levine, C., \& Samis, S. (2012). Home alone: Family caregivers providing complex chronic care. Washington, DC: AARP Public Policy Institute and United Hospital Fund.

Richard, L., Potvin, L., Kishchuk, N., Prlic, H., \& Green, L. W. (1996). Assessment of the integration of the ecological approach in health promotion programs. American Journal of Health Promotion, 10(4), 318-328.

Robison, J., Shugrue, N., Porter, M., Fortinsky, R.H., \& Curry, L.A. (2012). Transition from home care to nursing home: Unmet needs in a home- and community-based program for older adults. Journal of Aging \& Social Policy, 24, 251-270. 
Sanders, G. F., Fitzgerald, M. A., \& Bratteli, M. (2008). Mental health services for older adults in rural areas: An ecological systems approach. Journal of Applied Gerontology, 27(3), 252-266.

Simmons, S.F., Cadogan, M.P., Cabrera, G.R., Al-Samarrai, N.R., Jorge, J.S., Levy-Storms, L., Osterweil, D., \& Schnelle, J.F. (2004). The Minimum Data Set depression quality indicator: Does it reflect differences in care processes? The Gerontologist, 44(4), 554564.

Simon, S.E., Lipson, D.J., \& Stone, C.M. (2011). Mental disorders among non-elderly nursing home residents. Journal of Aging \& Social Policy, 23, 58-72.

U.S. Department of Health and Human Services. (1999). Older adults and mental health. In Mental health: A report of the surgeon general (pp. 335-401). Rockville, MD: Author.

Watts, M.O. (2013). Money Follows the Person: A 2012 survey of transitions, services, and costs. Washington, DC: The Kaiser Commission on Medicaid and the Uninsured.

Wenzlow, A. T., \& Lipson, D. J. (2009). Transitioning Medicaid enrollees from institutions to the community: Number of people eligible and number of transitions targeted under MFP. Ann Arbor, MI: Mathematica Policy Research. 


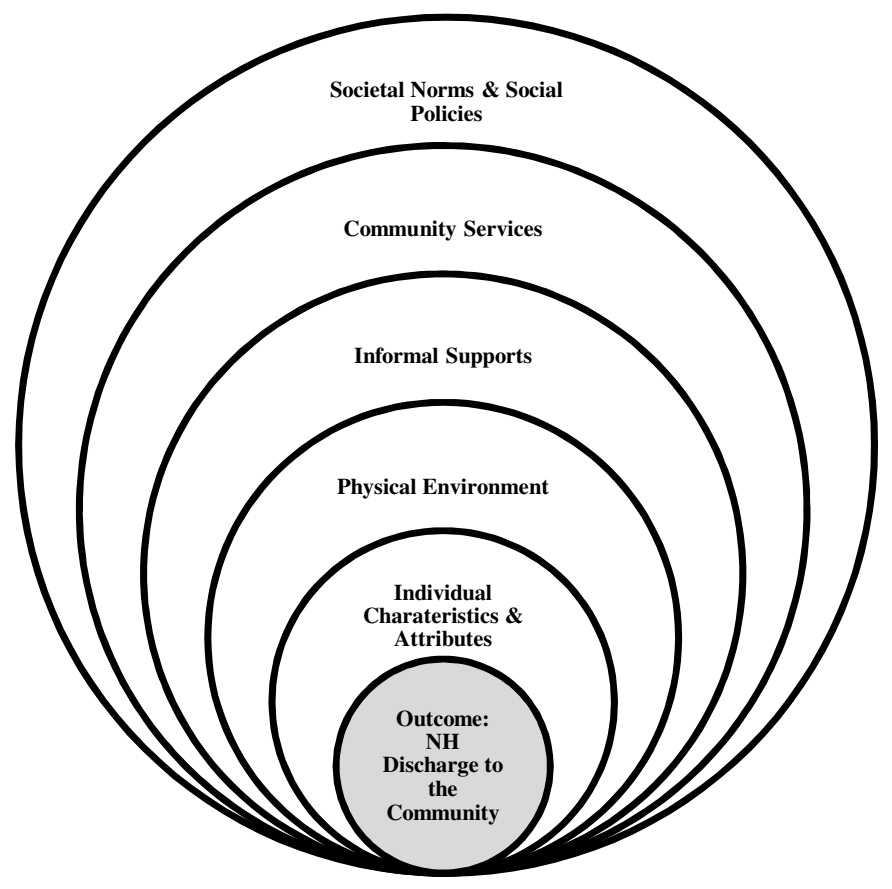

Figure 1. Ecological model for NH discharge to the community 


\section{Table 1. Health Characteristics}

\begin{tabular}{lr}
\hline Total Sample $(N=720)$ & $n(\%)$ \\
\hline Cognitive status (Cognitive Performance Scale) & \\
Very Severe Impairment & $11(1.5)$ \\
Severe Impairment & $0(0.0)$ \\
Moderately Severe Impairment & $126(17.5)$ \\
Moderate Impairment & $107(14.9)$ \\
Mild Impairment & $202(28.1)$ \\
Borderline Intact & $270(37.5)$ \\
Intact & \\
Diagnoses & $97(13.5)$ \\
Alzheimer's Disease or Other Dementia & $192(26.7)$ \\
Arthritis & $242(33.6)$ \\
COPD/Emphysema & $265(36.8)$ \\
Diabetes Mellitus & $80(11.1)$ \\
Cardiovascular Accident (Stroke) & $451(62.6)$ \\
Hypertension & $50(6.9)$ \\
Mental Retardation/Developmental Disability & \\
Chronic Disease Co-morbidity $(M=1.7, S D=1.1$, Range 0-5) & $259(36.0)$ \\
\hline Fall in Past 30 Days & \\
Medication Use $(M=13.4, S D=5.6$, Range=0-46) & $300(41.7)$ \\
Antipsychotics (1-7 days/week) & $349(48.5)$ \\
Antianxiety (1-7 days/week) & \\
\hline Note. Chronic Disease Co-morbidity includes these chronic diseases: arthritis, COPD \\
Emphysema, Diabetes mellitus, Cardiovascular accident (stroke), and hypertension.
\end{tabular}




\section{Footnotes}

${ }^{1}$ At one $\mathrm{NH}$, two discharge staff members were interviewed together. Thus, the total number of NH staff member participants is 11 , while the total number of interviews and NHs is 10 .

${ }^{2}$ All eligible individuals who had an admission assessment were included, regardless of their length of stay in the $\mathrm{NH}$. Thus, there were three individuals with an $\mathrm{NH}$ stay of 0 days indicating an admission and discharge on the same day.

${ }^{3} 105$ individuals with record of $\mathrm{NH}$ admission prior to July 1, 2005 were excluded from the patterns analysis. 